\title{
Isocratic Resolution of Fluoroquinolone-Based Antibiotics on the Phenylethyl-Bonded Phase under Nonaqueous Elution: A Consideration of the Separation Mechanism
}

\author{
Yu-Xuan Gao and Shushi Chen $\mathbb{D}$ \\ Department of Applied Chemistry, National Chiayi University, Chiayi 600, Taiwan \\ Correspondence should be addressed to Shushi Chen; schenphd@mail.ncyu.edu.tw
}

Received 14 November 2017; Accepted 5 March 2018; Published 31 May 2018

Academic Editor: Francesco Palmisano

Copyright (C) 2018 Yu-Xuan Gao and Shushi Chen. This is an open access article distributed under the Creative Commons Attribution License, which permits unrestricted use, distribution, and reproduction in any medium, provided the original work is properly cited.

\begin{abstract}
This paper reports the isocratic resolution of 10 fluoroquinolone-based antibiotics and their precursors on the phenylethyl-bonded phase under the elution of the nonaqueous mobile phase composed of acetonitrile, methanol, acetic acid, and triethylamine. Most of the analytes were baseline resolved within 10 minutes. The interaction simulation and Fourier-transform infrared spectroscopy (FTIR) data indicated that the carbonyl-containing group, a secondary or tertiary amine of an analyte, was heavily involved in the retention, resulting in retention with residual silanol groups on the stationary phase. In some cases, the elution reversal or resolution enhancement of analytes was observed when the volume of acidic or basic additive in the mobile phase was dominant. However, the $\pi-\pi$ complexation interaction between the fluorine-attached phenyl group of the analyte and the phenylethyl moiety on the stationary phase was not observed. Consequently, the resolution could not be reproduced either on the other stationary phase modified with $\mathrm{C}_{18}$, phenyl, or phenylhexyl moiety under the same chromatographic conditions or under the aqueous elution.
\end{abstract}

\section{Introduction}

Quinolone-based antibiotics are universally effective and used extensively against Gram-negative bacteria in livestock and humans to treat a wide variety of diseases [14]. Because fluoroquinolones and their precursors are only partially metabolized after administration, these compounds are discharged into municipal wastewater and environmental aquatic systems. Antibiotic pollution in agricultural crops is expected if the crops are irrigated with these water resources and fertilized with sewage sludge from water treatment plants or livestock manure. For environmental and public safety, several methods have been developed for high-performance liquid chromatography (HPLC) analysis and the recovery of these antibiotics from wastewater and soil using adsorbents [5-17]. The HPLC approach in these studies often involved gradient or isocratic elution combined with a $\mathrm{C}_{18}$ stationary phase to resolve a limited number of antibiotics. However, an inconsistent elution order is often observed under the aqueous chromatographic condition [10,15-17].
In this study, several stationary phases, including phenyl $\left(\mathrm{C}_{\mathrm{ph}}\right)$, phenylethyl $\left(\mathrm{C}_{\mathrm{phe}}\right)$, phenylhexyl $\left(\mathrm{C}_{\mathrm{phh}}\right), \mathrm{C}_{8}$, and $\mathrm{C}_{18}$ were examined to optimize the resolution of 10 fluoroquinolone-based antibiotics and their precursors under isocratic nonaqueous elution. The resolution was further optimized by altering the volume of acidic or basic additive in the mobile phase. Under optimized conditions, Fouriertransform infrared spectroscopy (FTIR) data were collected to explore the resolution mechanism. In addition, interaction simulation was performed to gain a theoretical understanding of the mechanism leading to the resolution.

\section{Experimental Procedures}

2.1. Apparatus. An HPLC system (Model L-7100, Tokyo, Japan) coupled with a D-2500 chromatopac data station (Shimadzu, Kyoto, Japan) and ultraviolet (UV) detector with the detection wavelength set at $294 \mathrm{~nm}$ was used for resolution optimization under nonaqueous elution. Columns packed 


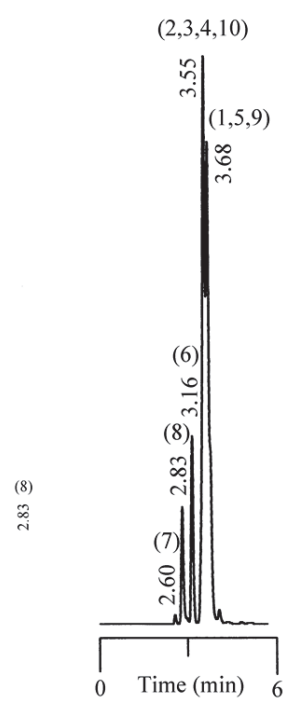

(a)

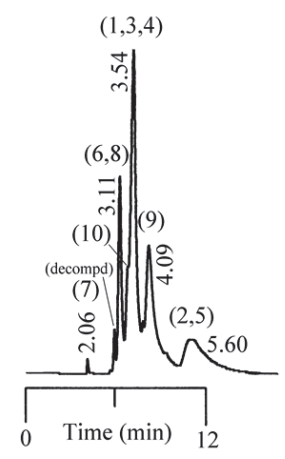

(b)

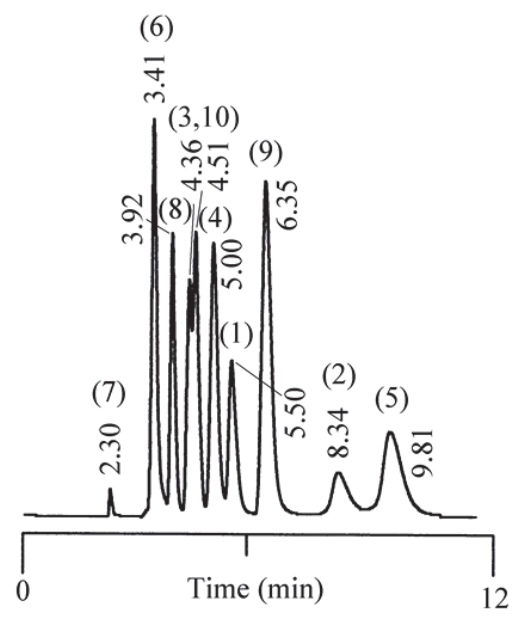

(c)

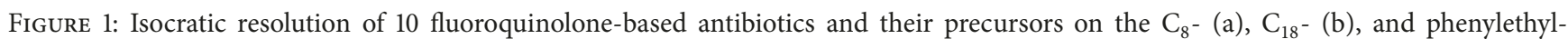
bonded stationary phases under optimized elution of nonaqueous mobile phases of 490/10/1/1, 500/0/1/3, and 490/10/1/2 by volume (acetonitrile/methanol/acetic acid/triethylamine, $v / v$ ), respectively. The numbering system of compounds from left to right is as follows: piroxicam (7), nalidixic acid (6), cinoxacin (8), difloxacin (3), enrofloxacin (10), pefloxacin (4), ofloxacin (1), lomefloxacin (9), ciprofloxacin (2), and pipemidic acid (5).

with $\mathrm{C}_{8^{-}}, \mathrm{C}_{18^{-}}$, phenyl-, phenylethyl-, and phenylhexylmodified silica gels $(250 \times 4.6 \mathrm{~mm}$ internal diameter; $5 \mu \mathrm{m}$ particle diameter) were manufactured by GL Sciences Inc. (Tokyo, Japan) and used for HPLC resolution at a flow rate of $1.0 \mathrm{~mL} / \mathrm{min}$. The mobile phase for HPLC elution was a mixture of acetonitrile, methanol, glacial acetic acid, and triethylamine.

FTIR spectra were obtained by scanning samples 10 times on a Shimadzu Model FTIR-8400 system at a resolution of $4 \mathrm{~cm}^{-1}$. In the FTIR measurements, a small fraction of the stationary phase in the column purchased was sampled and immersed in the mobile phase containing the analyte and then collected, pelleted with $\mathrm{KBr}$ after being washed with purified water, and dried.

2.2. Chemicals. All chemicals used in this study, including the fluoroquinolone-based antibiotics and their precursors, were purchased from Sigma (St. Louis, MO, USA) or Aldrich (Milwaukee, WI, USA). All HPLC-grade solvents (acetonitrile, methanol, glacial acetic acid, and triethylamine) were obtained from Fisher Scientific (Pittsburgh, PA, USA) and Merck Taiwan Ltd. (Taipei, Taiwan, ROC). In all cases, filtered $(0.2 \mathrm{~mm})$ and distilled water was used. Antibiotic standards were purchased to identify the chromatographic peaks.

\subsection{Theoretical Computational Calculation with Spartan'14} Software. A theoretical calculation for single point energy was conducted according to a semiempirical molecular orbital calculation method (Parameterized Model 3) by using Spartan'14 software from Wavefunction, Inc. (Irvine, CA, USA). Atoms on the stationary phase and analyte were simulated to interact with one another to determine the lowest formation energy at the ground state (i.e., the heat of formation). Stationary phase moieties including $\mathrm{C}_{8}, \mathrm{C}_{18}$, phenyl, phenylethyl, and phenylhexyl placed around the fixed position analyte were considered in the evaluation. Prior to the calculation, the molecular energy was first minimized by modifying the bond lengths and angles until a minimumenergy conformer was found. The mutual distance between the analyte and stationary phase moiety was subsequently altered as a result.

\section{Results and Discussion}

3.1. Stationary Phase Consideration. Tables 1 and 2 summarize the chromatographic data for the resolution of nine selected fluoroquinolone-based antibiotics and their precursors on the phenylethyl-bonded phase under the isocratic elution of various nonaqueous mobile phases with the predominantly acidic or basic additive by volume, respectively. A typical chromatogram showing the resolution of 10 analytes under optimized conditions on the phenylethyl-boned stationary phase within 10 minutes is shown in Figure 1(c). Under the same conditions, the resolution was not reproducible on stationary phases such as $\mathrm{C}_{8}$ (a) and $\mathrm{C}_{18}$ (b) after comparison. Evidently, analytes were less retained and poorly resolved on the hydrocarbon-typed stationary phases, and the peak tailing for certain analytes strongly suggests that the hydrophobicity-oriented approach (e.g., $\mathrm{C}_{8}$ and $\mathrm{C}_{18}$ phases) toward the resolution is impractical. Upon close examination of these chromatograms, elution reversal for several analytes was observed on $\mathrm{C}_{8}$ but not on $\mathrm{C}_{18}$. This was evident in the resolution of ofloxacin and ciprofloxacin on $\mathrm{C}_{8}$ and $\mathrm{C}_{\text {phe }}$ phases under the elution of $\mathrm{ACN} / 1 / 3$ by volume 


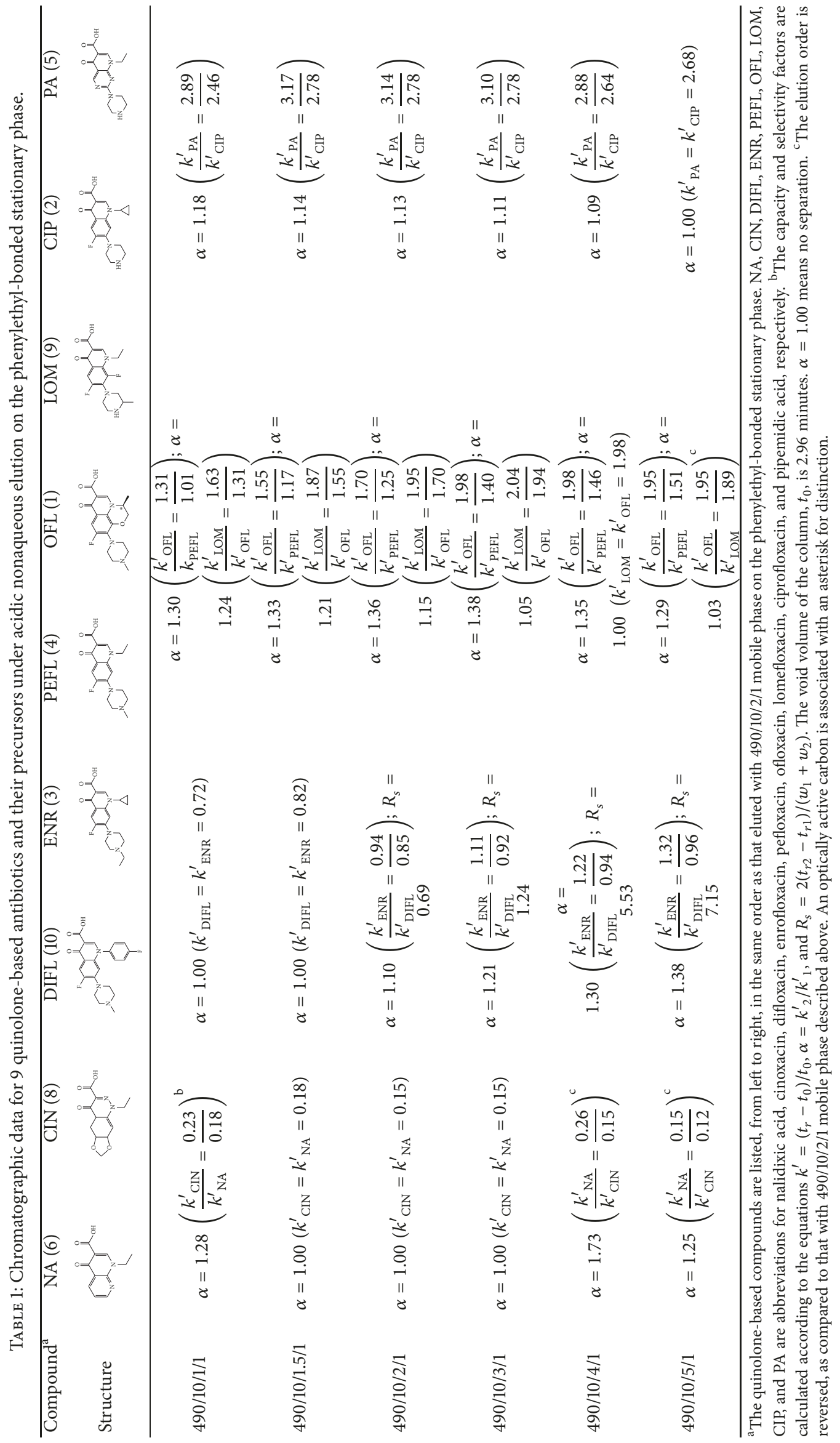




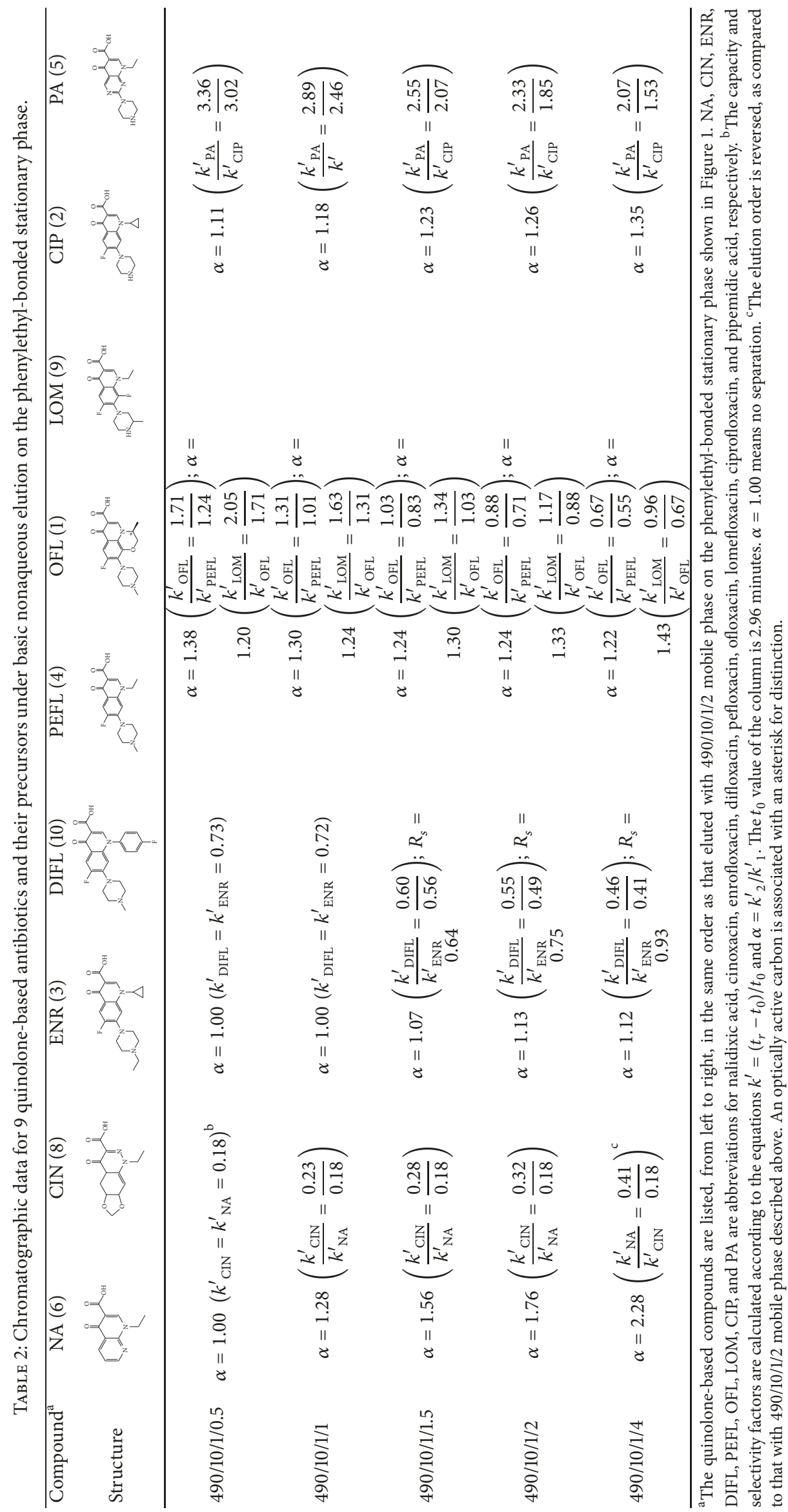




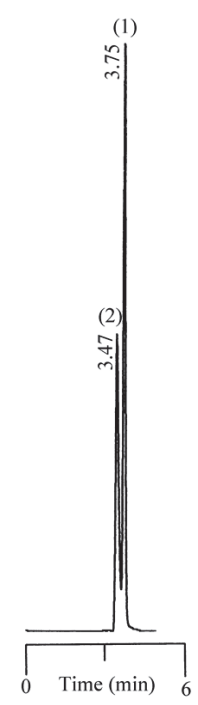

(a)

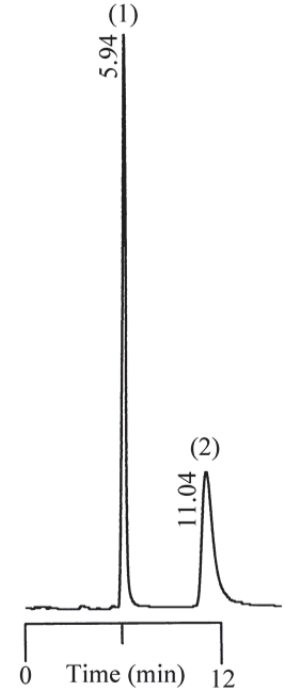

(b)

FIGURE 2: Resolution of ofloxacin and ciprofloxacin under the elution of ACN/1/3 by volume (acetonitrile/acetic acid/triethylamine, $v / v$ ) on the $\mathrm{C}_{8}$ (a) and phenylethyl (b) stationary phases. Note that the elution order has been reversed. The numbering system is the same as that in Figure 1.

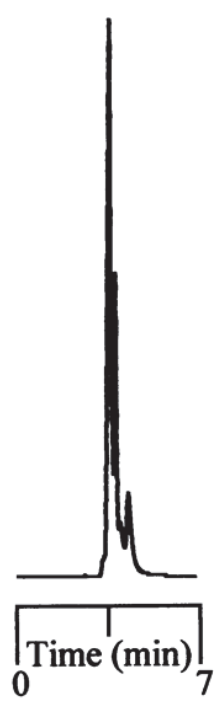

(a)

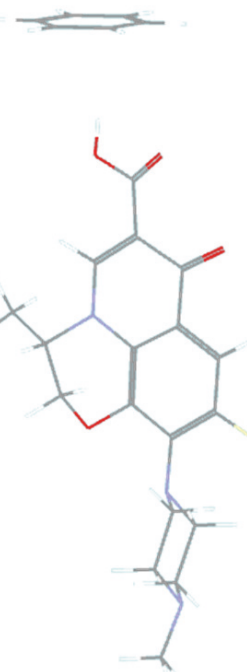

(b)
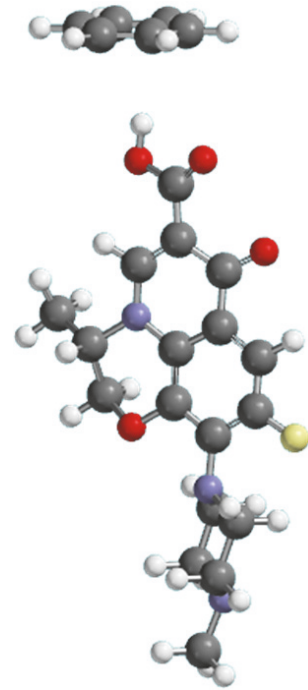

(c)

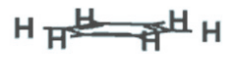

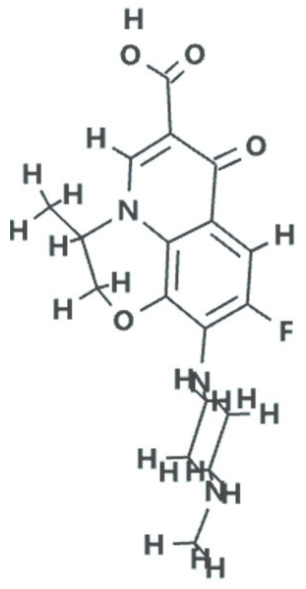

(d)

FIGURE 3: Resolution of 10 fluoroquinolone-based antibiotics and their precursors on the phenyl-bonded stationary phase under the elution of nonaqueous mobile phase of $490 / 10 / 1 / 2$ by volume (acetonitrile/methanol/acetic acid/triethylamine, $v / v$ ) (a). The interaction simulation between ofloxacin and phenyl moiety expressed in stereochemistry molecular (b) and stick and ball (c) models and molecular structure (d) for easy comparison.

(acetonitrile/acetic acid/triethylamine, $v / v$ ), as shown in Figure 2. The discrepancies in the chromatographic profile and elution order results strongly suggest that the hydrophobic interaction was not the only force responsible for resolution on the $\mathrm{C}_{\text {phe }}$ phase.

Phenyl- and phenylhexyl-modified silica gels were two other stationary phases examined in this study to further explore the involvement of $\pi-\pi$ complexation in retention and, consequently, resolution under nonaqueous elution.
Figure 3(a) shows the resolution of analytes on the phenylbonded stationary phase under the same elution of the nonaqueous mobile phase of $490 / 10 / 1 / 2$ by volume (acetonitrile/methanol/acetic acid/triethylamine, $v / v$ ). Notably, the only difference between the two stationary phases was in the alkyl group linking the aromatic moiety to the silica gel. However, the profile of the chromatogram, which was characterized by poor resolution and a short retention scale, was totally different from that in Figure 1(c) after comparison, 


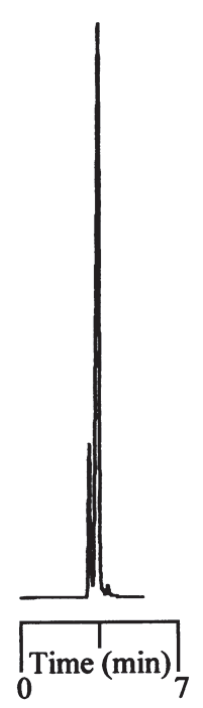

(a)

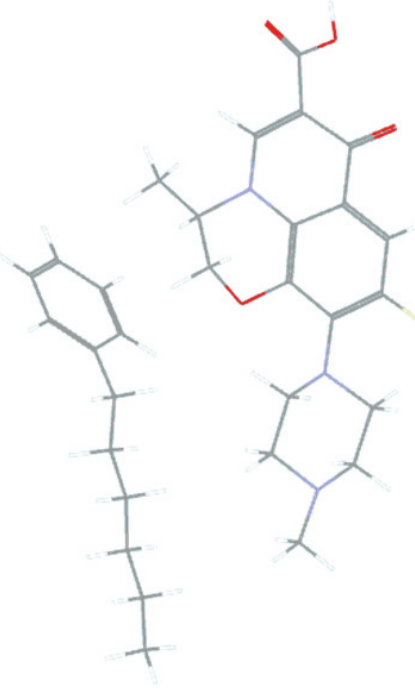

(b)

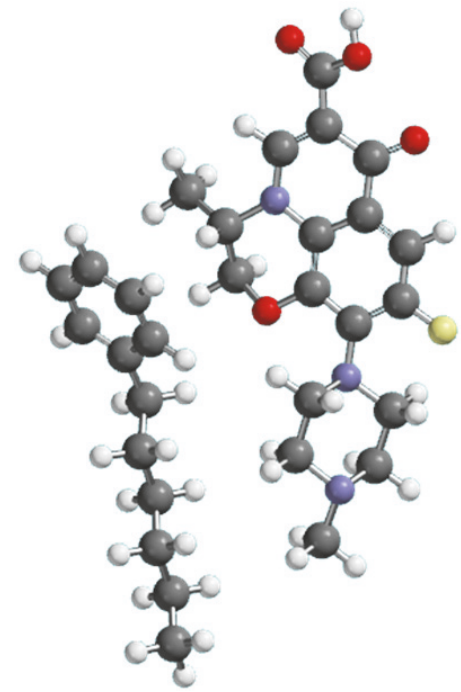

(c)<smiles>[H][Z]([H])([H])[C@H]([CH])[C@H]([CH])c1ccccc1</smiles>

(d)

FIGURE 4: Resolution of 10 fluoroquinolone-based antibiotics and their precursors on the phenylhexyl-bonded stationary phase under the elution of nonaqueous mobile phase of $490 / 10 / 1 / 2$ by volume (acetonitrile/methanol/acetic acid/triethylamine, $v / v$ ) (a). The interaction simulation between ofloxacin and phenylhexyl moiety expressed in stereochemistry molecular (b) and stick and ball (c) models and molecular structure (d) for easy comparison.

indicating that the $\pi-\pi$ complexation interaction was not the main force contributing to the resolution. In the interaction simulation between ofloxacin and the phenyl moiety (Figures $3(\mathrm{~b}), 3(\mathrm{c})$, and $3(\mathrm{~d})$ ), the same conclusion was reached based on a lack of observable $\pi-\pi$ stacking complexation. Notably, these two conformers exhibited the most stable conformation among themselves and seven others (Table 3 ) when assessed based on the amount of energy released upon association. In the case of $\mathrm{C}_{\mathrm{phh}}$, a phase possessing a long hydrocarbon linker, the resolution in Figure 4(a) showed no improvement under the same mobile phase elution. However, the hexyl hydrocarbon linker of the phase did contribute a degree of retention based on the simulation results in Figures 4(b), $4(c)$, and $4(d)$, similar to the $C_{8}$ and $C_{18}$ phases discussed previously. In addition, there was no evidence of $\pi-\pi$ stacking complexation in this most stable conformation.

3.2. Interaction Simulation and FTIR Data. Except in the case of ofloxacin, several analytes were examined in the interaction simulation with the three aforementioned stationary phases. The results listed in Table 3 indicate that generally the $\mathrm{C}_{\mathrm{phh}}$ and $\mathrm{C}_{\mathrm{ph}}$ phases released the most and least energy, respectively, from association with the analytes. Additionally, the number for the amount of energy released within the range specified in Table 3 was the greatest in the $\mathrm{C}_{\mathrm{phh}}$ phase. All simulation results should lead to a significant retention scale because of the intensive interactions of two conformers and thus should lead to the possibility of improving the resolution on the $\mathrm{C}_{\text {phh }}$ phase. However, the resolution on these phases under the same chromatographic conditions was observed to be incomparable with that on the $\mathrm{C}_{\text {phe }}$ phase, strongly suggesting that there was some form of force other than hydrophobicity or $\pi-\pi$ complexation interaction involved in the chromatographic process. Based on the FTIR data, residual silanol groups on the surface of the silica gel were considered responsible for producing the strong dipoledipole interaction. Upon close examination of the spectra for three selected analytes with the $\mathrm{C}_{\text {phe }}$ phase in Figure 5(a), the stretching vibrations centered at $3463.72 \mathrm{~cm}^{-1}$ for the $-\mathrm{OH}$ or -NH functional group on the $\mathrm{C}_{\text {phe }}$ phase were all red shifted on a large scale because of the conformational interaction compared with that of the $\mathrm{C}_{18}$ phase (Figure 5(b)) under the same experimental conditions. Note that the acidity of both analyte and silanol group and thus the dipole-dipole interaction are enhanced in acetonitrile [18]. However, the enhancement would not be observed under the elution of aqueous mobile phase due to the competition interaction with silanol group from water molecule. Conversely, the shift of $\mathrm{CH}_{3}$ and $\mathrm{CH}_{2}$ stretching (symmetric and asymmetric) on the $\mathrm{C}_{18}$ phase was insignificant after association, indicating that the hydrophobic interaction between the analyte and $\mathrm{C}_{18}$ molecule was not the major force contributing to the retention on the phase. The peaks centered at 1640.15 and $1643.33 \mathrm{~cm}^{-1}$ in Figure 5 were assigned to the residual water molecule bending vibration on $\mathrm{C}_{18}$ and $\mathrm{C}_{\text {phe }}$ phases, respectively. The difference in bending frequency between these two phases was due to the environmental variation surrounding the water molecule. The environmental variation was further complicated as the analyte was near the phase molecule and interacting with it, which resulted in an apparent red shift in frequency. By contrast, the vibrational frequency of the Si-OSi backbone network (centered at 1098.47 and $1095.14 \mathrm{~cm}^{-1}$ on $\mathrm{C}_{18}$ and $\mathrm{C}_{\text {phe }}$ phases, respectively) on both examined phases was not altered upon the association of the analyte and modifier molecule of the stationary phase. These FTIR results suggest that the interaction occurred near the surface, 


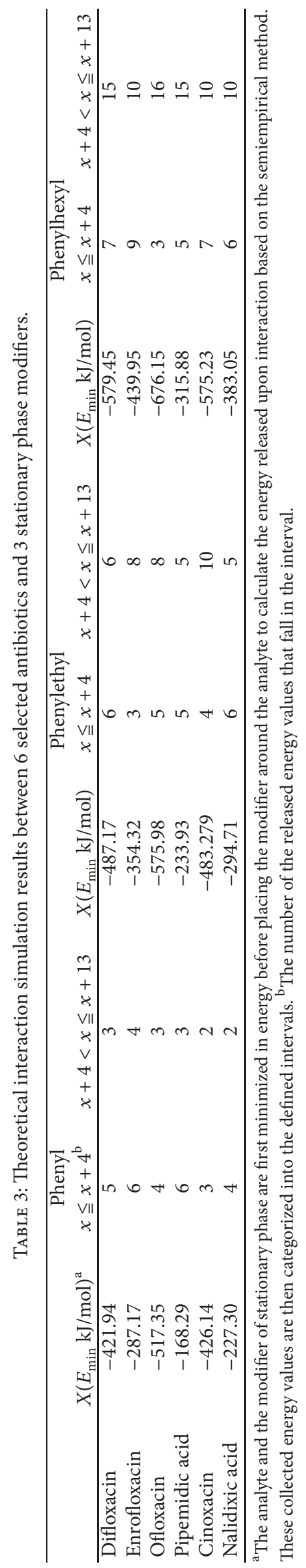




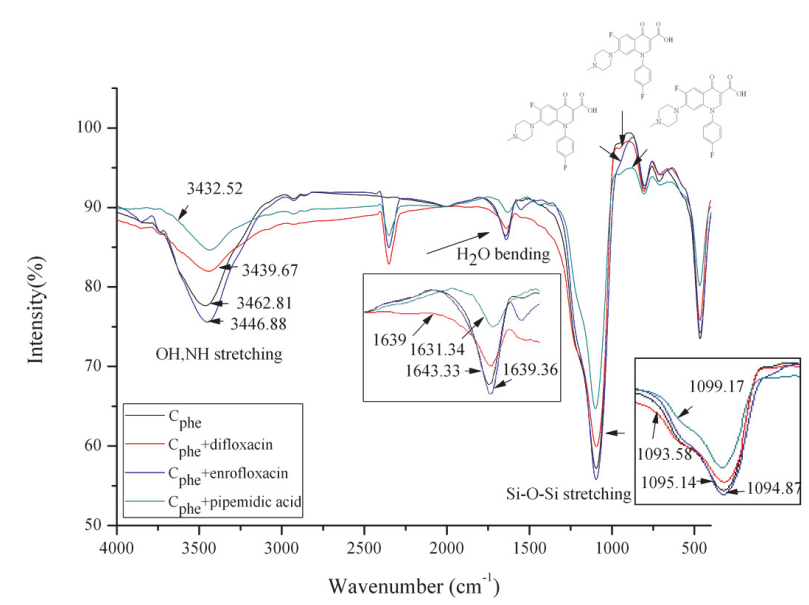

(a)

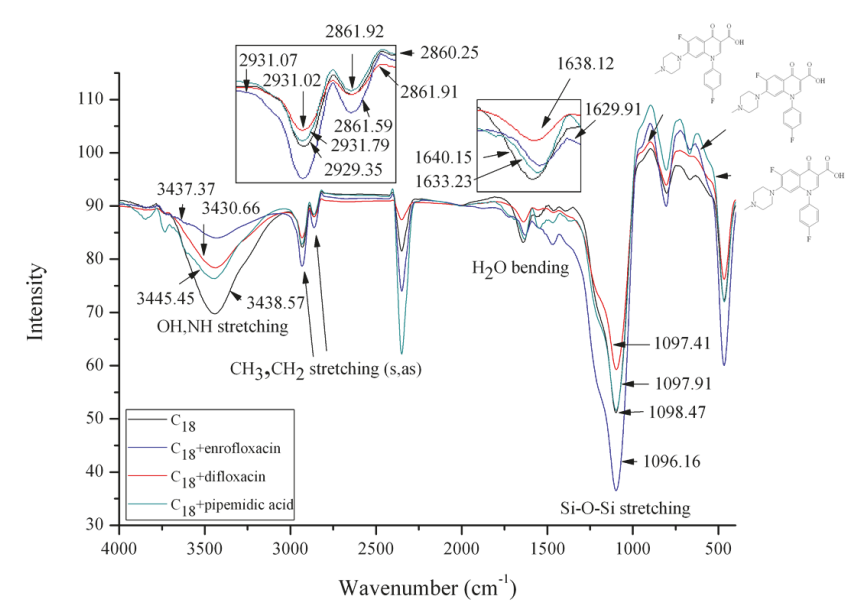

(b)

FIGURE 5: Superimposed FTIR spectra for three selected analytes, including difloxacin, enrofloxacin, and pipemidic acid on the phenylethyl (a) and $\mathrm{C}_{18}$ (b) phases. In both cases, FTIR spectra for phenylethyl and $\mathrm{C}_{18}$ phases were included for comparison.

and thus the peak tailing that resulted from penetration of the analyte molecule deep into the silica matrix was not observed.

\subsection{Effect of Acidic and Basic Additives on Retention, Elution} Order, and Resolution. The chromatographic data in Tables 1 and 2 for the nine selected fluoroquinolone-based antibiotics and their precursors on phenylethyl-bonded phase under the isocratic elution of various nonaqueous mobile phases with predominantly acidic or basic additive by volume may provide experimental evidence of the interaction force contributing to resolution. Under the isocratic elution using the mobile phase with the predominantly acidic additive (Table 1), the capacity factor for all the examined analytes was increased with the volume of acidic additive. However, the selectivity factor could remain constant (e.g., pefloxacin versus ofloxacin) or become larger (e.g., difloxacin versus enrofloxacin) or smaller (e.g., ofloxacin versus lomefloxacin) in value, depending on how the capacity factor of the analyte is affected by the volume increment of acidic additive in the mobile phase. Notably, the resolution factor was enhanced considerably because of the improvement of the selectivity factor in the cases of difloxacin and enrofloxacin as the volume of acidic additive in the mobile phase was increased from 2 to $5 \mathrm{ml}$. In other words, difloxacin and enrofloxacin had much greater resolution values than those at baseline $\left(R_{s}=1.50\right)$ under the elution of the mobile phase 490/10/5/1 by volume (acetonitrile/methanol/acetic acid/triethylamine, $v / v$ ). Protonation of functional groups on the stationary phase and the analyte on a large scale enhanced the dipoledipole interaction, and thus the magnitude of this effect was believed to be structure dependent. Essentially, this conclusion is consistent with many previous reports dealing with $\mathrm{p} K_{\mathrm{a}}$ value determination for several quinolone and fluoroquinolone antibiotics in aqueous solutions [19-23].

The capacity factor for the resolution of all the examined analytes on the $\mathrm{C}_{\text {phe }}$ phase under isocratic elution using the mobile phase with the predominantly basic additive was decreased (Table 2), likely because of the deprotonation of the functional groups on the stationary phase and analyte. Notably, the capacity factor for the aforementioned analytes was observed to be very different; these analytes are usually retained to a lesser extent and thus eluted quickly. However, these analytes also had greater resolution even on the small retention scale. We also observed improvements in capacity factors, resulting in the enhancement of the resolution factor, similar to the case of enrofloxacin and difloxacin. Apparently, the enhancement of dipole-dipole interactions under the predominantly acidic additive was not generally advantageous to the resolution of fluoroquinolone-based antibiotics on the phenylethyl-bonded phase under nonaqueous elution.

Except for manipulating the capacity factor of analytes and avoiding possible hydrolysis of the stationary phase under nonaqueous conditions, it is possible to selectively reverse the elution order of some fluoroquinolone-based antibiotics and their precursors on the phenylethyl-bonded phase. This could be accomplished by adjusting rather than enhancing the degree of dipole-dipole interactions by altering the volume of acidic or basic additive in the mobile phase. Table 1 shows that the elution order of nalidixic acid and cinoxacin was reversed as the volume of acidic additive in the mobile phase was increased to $4 \mathrm{ml}$ or higher. Elution reversal was also observed in ofloxacin and lomefloxacin when the volume of acidic additive in the mobile phase was $5 \mathrm{ml}$. Table 2 shows that the reversed elution of nalidixic acid and cinoxacin was observed when the volume of basic additive in the mobile phase was increased to $4 \mathrm{ml}$. Notably, by switching the mobile phase from $490 / 10 / 1 / 2$ by volume (acetonitrile/methanol/acetic acid/triethylamine, $v / v$ ) to $490 / 10 / 2 / 1$, the elution of enrofloxacin and difloxacin was reversed. Upon close examination of the structure of these analytes, each analyte contained a tertiary amine attached to the moiety suitable for the creation of steric hindrance. 
However, some analytes such as lomefloxacin, ciprofloxacin, and pipemidic acid bore secondary amines for retention purposes only.

\section{Conclusion}

Under nonaqueous elution, the resolution of 10 fluoroquinolone-based antibiotics and their precursors on the phenylethyl-bonded phase was isocratically, rapidly, and efficiently performed within 10 minutes. Based on the interaction simulation and FTIR data, $\pi-\pi$ complexation and hydrophobicity were not the major forces contributing to this resolution. Instead, strong interactions between residual silanol groups on the silica gel were responsible.

Manipulating the capacity factor of analytes by altering the volume of acidic or basic additive in the mobile phase can reverse the elution order and improve the selectivity factor, thereby considerably enhancing the resolution factor of the analytes in some cases. These results would be very useful in determining these antibiotics in the dairy products under nonaqueous elution, which has been currently under investigation. In any case, avoiding possible hydrolysis of the stationary phase under nonaqueous elution conditions could be expected.

\section{Additional Points}

Under the same nonaqueous chromatographic conditions, the elution order was reversed as switching the stationary phase from phenylethyl- to $\mathrm{C}_{18}$-based. The elution reversal was also observed as altering the acid/base ratio in the mobile phase.

\section{Disclosure}

This article does not contain any studies with human participants or animals performed by any of the authors.

\section{Conflicts of Interest}

The authors declare no conflicts of interest.

\section{Acknowledgments}

This study was funded by the Ministry of Science and Technology (MST), Taiwan, under Grant no. 106-2113-M-415005 .

\section{Supplementary Materials}

The interaction simulation after energy minimization between ofloxacin and $\mathrm{C}_{18}$ moiety expressed in stereochemistry molecular (left) and stick and ball (right) models for easy comparison. The hydrophobic interaction is clearly dominated in the simulation. (Supplementary Materials)

\section{References}

[1] J. S. Wolfson and D. C. Hooper, "Fluoroquinolone antimicrobial agents," Clinical Microbiology Reviews, vol. 2, no. 4, pp. 378-424, 1989.
[2] D. E. King, R. Malone, and S. H. Lilley, "New classification and update on the quinolone antibiotics," American Family Physician, vol. 61, no. 9, pp. 2741-2748, 2000.

[3] A. Fitton, "The quinolones: an overview of their pharmacology," Clinical Pharmacokinetics, vol. 22, no. 1, pp. 1-11, 1992.

[4] G. E. Stein, "Pharmacokinetics and pharmacodynamics of newer fluoroquinolones," Clinical Infectious Diseases, vol. 23, no. 1, pp. S19-S24, 1996.

[5] T. Zhao, X. Guan, W. Tang, Y. Ma, and H. Zhang, "Preparation of temperature sensitive molecularly imprinted polymer for solid-phase microextraction coatings on stainless steel fiber to measure ofloxacin," Analytica Chimica Acta, vol. 853, no. 1, pp. 668-675, 2015.

[6] E. Turiel, A. Martín-Esteban, and J. L. Tadeo, "Multiresidue analysis of quinolones and fluoroquinolones in soil by ultrasonic-assisted extraction in small columns and HPLCUV," Analytica Chimica Acta, vol. 562, no. 1, pp. 30-35, 2006.

[7] A. Speltini, M. Sturini, F. Maraschi, L. Consoli, A. Zeffiro, and A. Profumo, "Graphene-derivatized silica as an efficient solid-phase extraction sorbent for pre-concentration of fluoroquinolones from water followed by liquid-chromatography fluorescence detection," Journal of Chromatography A, vol. 1379, pp. 9-15, 2015.

[8] K. He and L. Blaney, "Systematic optimization of an SPE with HPLC-FLD method for fluoroquinolone detection in wastewater," Journal of Hazardous Materials, vol. 282, pp. 96105, 2015.

[9] R. Mirzajani and F. Kardani, "Fabrication of ciprofloxacin molecular imprinted polymer coating on a stainless steel wire as a selective solid-phase microextraction fiber for sensitive determination of fluoroquinolones in biological fluids and tablet formulation using HPLC-UV detection," Journal of Pharmaceutical and Biomedical Analysis, vol. 122, pp. 98-109, 2016.

[10] A. Espinosa-Mansilla, A. Muñoz De La Peña, D. González Gómez, and F. Salinas, "HPLC determination of enoxacin, ciprofloxacin, norfloxacin and ofloxacin with photoinduced fluorimetric (PIF) detection and multiemission scanning: Application to urine and serum," Journal of Chromatography B, vol. 822, no. 1-2, pp. 185-193, 2005.

[11] N. Cavazos-Rocha, I. Carmona-Alvarado, L. Vera-Cabrera, N. Waksman-De-torres, and M. De La Luz Salazar-Cavazos, "HPLC method for the simultaneous analysis of fluoroquinolones and oxazolidinones in plasma," Journal of Chromatographic Science (JCS), vol. 52, no. 10, pp. 1281-1287, 2014.

[12] V. F. Samanidou, C. E. Demetriou, and I. N. Papadoyannis, "Direct determination of four fluoroquinolones, enoxacin, norfloxacin, ofloxacin, and ciprofloxacin, in pharmaceuticals and blood serum by HPLC," Analytical and Bioanalytical Chemistry, vol. 375, no. 5, pp. 623-629, 2003.

[13] M. Ramos-Payán, M. Villar-Navarro, R. Fernández-Torres, M. Callejón-Mochón, and M. Á. Bello-López, "Electromembrane extraction (EME) - An easy, novel and rapid extraction procedure for the HPLC determination of fluoroquinolones in wastewater samples," Analytical and Bioanalytical Chemistry, vol. 405, no. 8, pp. 2575-2584, 2013.

[14] D. Ašperger, V. Tišler, and M. Zrnčić, "HPLC-DAD-FLD determination of veterinary pharmaceuticals in pharmaceutical industry wastewater with precolumn derivatization using fluorescamine," Chromatographia, vol. 77, no. 15, pp. 1059-1066, 2014. 
[15] S. S. Bozkurt, D. Erdogan, M. Antep, N. Tuzmen, and M. Merdivan, "Use of ionic liquid based chitosan as sorbent for preconcentration of fluoroquinolones in milk, egg, fish, bovine, and chicken meat samples by solid phase extraction prior to HPLC determination," Journal of Liquid Chromatography \& Related Technologies, vol. 39, no. 1, pp. 21-29, 2016.

[16] G. N. Wang, C. Feng, H. C. Zhang, Y. Q. Zhang, L. Zhang, and J. P. Wang, "Determination of fluoroquinolone drugs in meat by ionic-liquid-based dispersive liquid-liquid microextractionhigh performance liquid chromatography," Analytical Methods, vol. 7, no. 3, pp. 1046-1052, 2015.

[17] Y.-K. Lv, L. Yang, X.-H. Liu, Z.-Y. Guo, and H.-W. Sun, "Preparation and evaluation of a novel molecularly imprinted hybrid composite monolithic column for on-line solid-phase extraction coupled with HPLC to detect trace fluoroquinolone residues in milk," Analytical Methods, vol. 5, no. 7, pp. 1848-1855, 2013.

[18] H. A. Laitinen and W. E. Harris, Chemical Analysis, McGrawHill, Inc., New York, NY, USA, 2nd edition, 1975.

[19] E. Jiménez-Lozano, I. Marqués, D. Barrón, J. L. Beltrán, and J. Barbosa, "Determination of pKa values of quinolones from mobility and spectroscopic data obtained by capillary electrophoresis and a diode array detector," Analytica Chimica Acta, vol. 464, no. 1, pp. 37-45, 2002.

[20] A. I. Drakopoulos and P. C. Ioannou, "Spectrofluorimetric study of the acid-base equilibria and complexation behavior of the fluoroquinolone antibiotics ofloxacin, norfloxacin, ciprofloxacin and pefloxacin in aqueous solution," Analytica Chimica Acta, vol. 354, no. 1-3, pp. 197-204, 1997.

[21] M. Shalaeva, J. Kenseth, F. Lombardo, and A. Bastin, "Measurement of dissociation constants ( $\mathrm{p} K_{a}$ values) of organic compounds by multiplexed capillary electrophoresis using aqueous and cosolvent buffers," Journal of Pharmaceutical Sciences, vol. 97, no. 7, pp. 2581-2606, 2008.

[22] M. Shimizu, Y. Takase, S. Nakamura et al., "Pipemidic acid, a new antibacterial agent active against pseudomonas aeruginosa: in vitro properties," Antimicrobial Agents and Chemotherapy, vol. 8, no. 2, pp. 132-138, 1975.

[23] O. Lorphensri, J. Intravijit, D. A. Sabatini, T. C. G. Kibbey, K. Osathaphan, and C. Saiwan, "Sorption of acetaminophen, $17 \alpha$-ethynyl estradiol, nalidixic acid, and norfloxacin to silica, alumina, and a hydrophobic medium," Water Research, vol. 40, no. 7, pp. 1481-1491, 2006. 

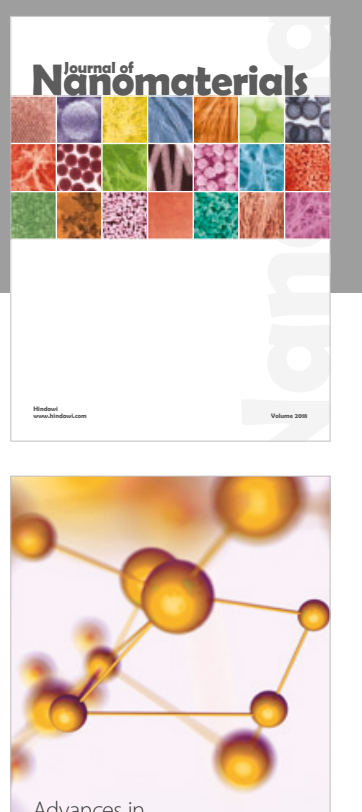

Physical Chemistry
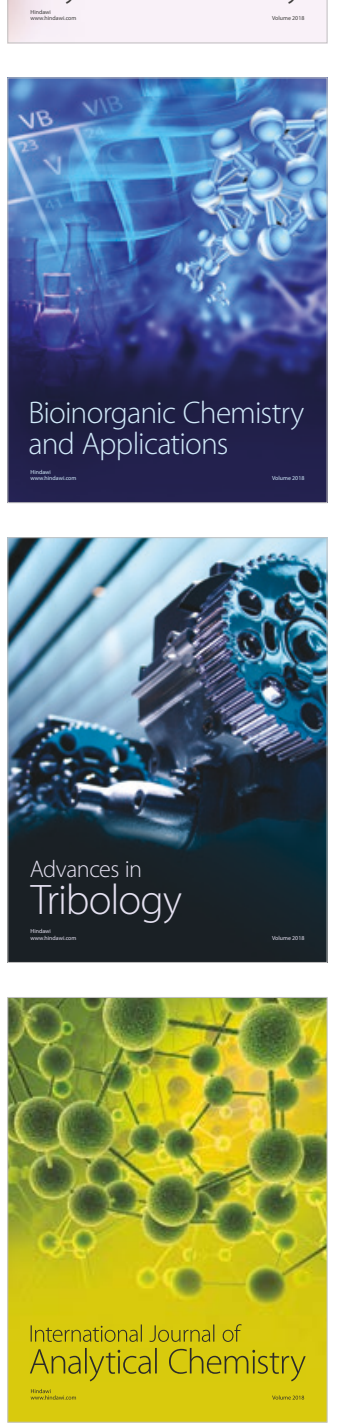

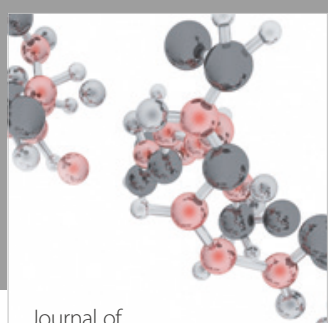

Analytical Methods

in Chemistry

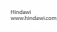

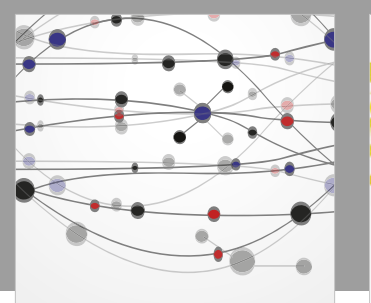

The Scientific World Journal

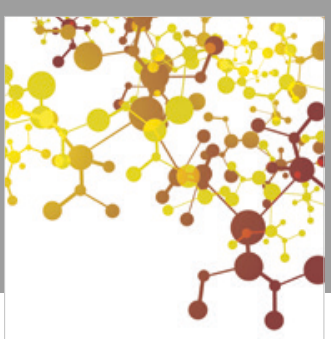

Journal of

Applied Chemistry
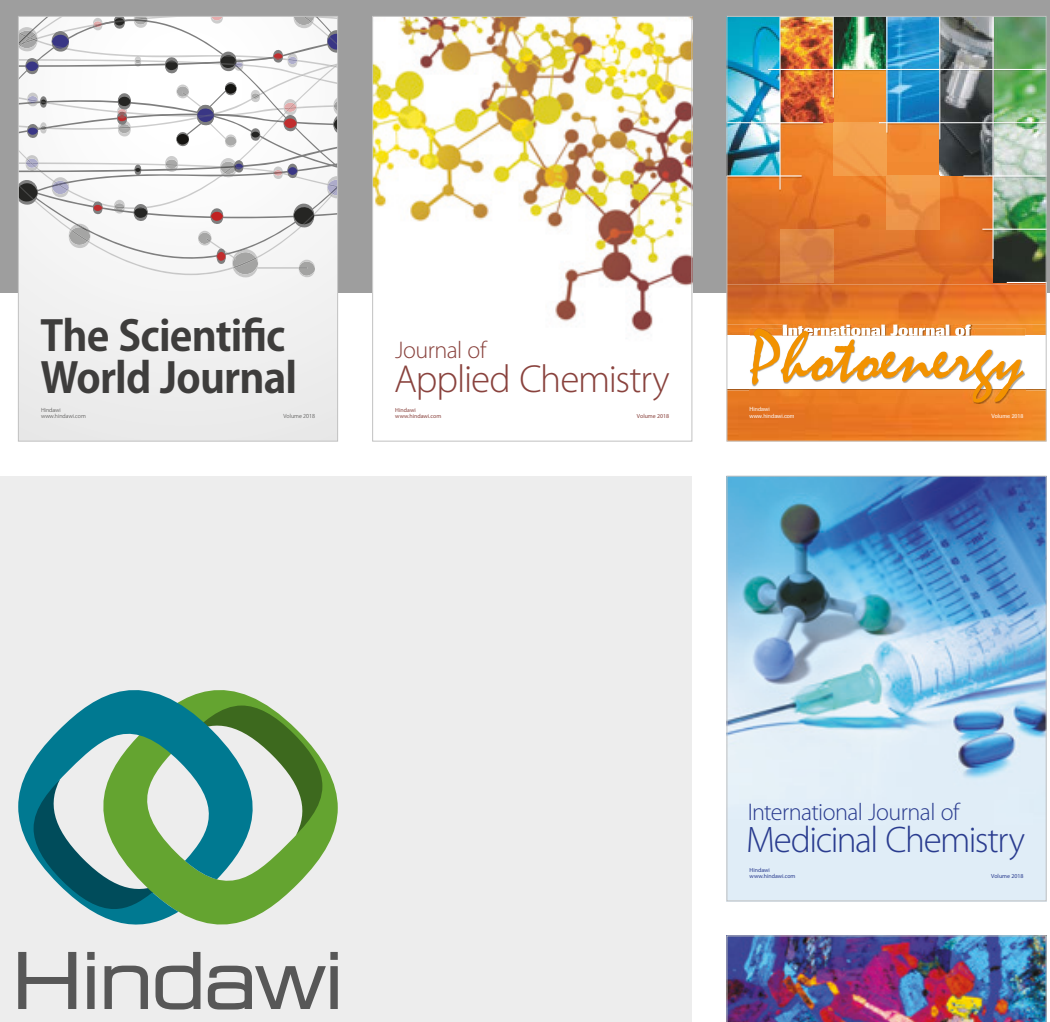

Submit your manuscripts at

www.hindawi.com
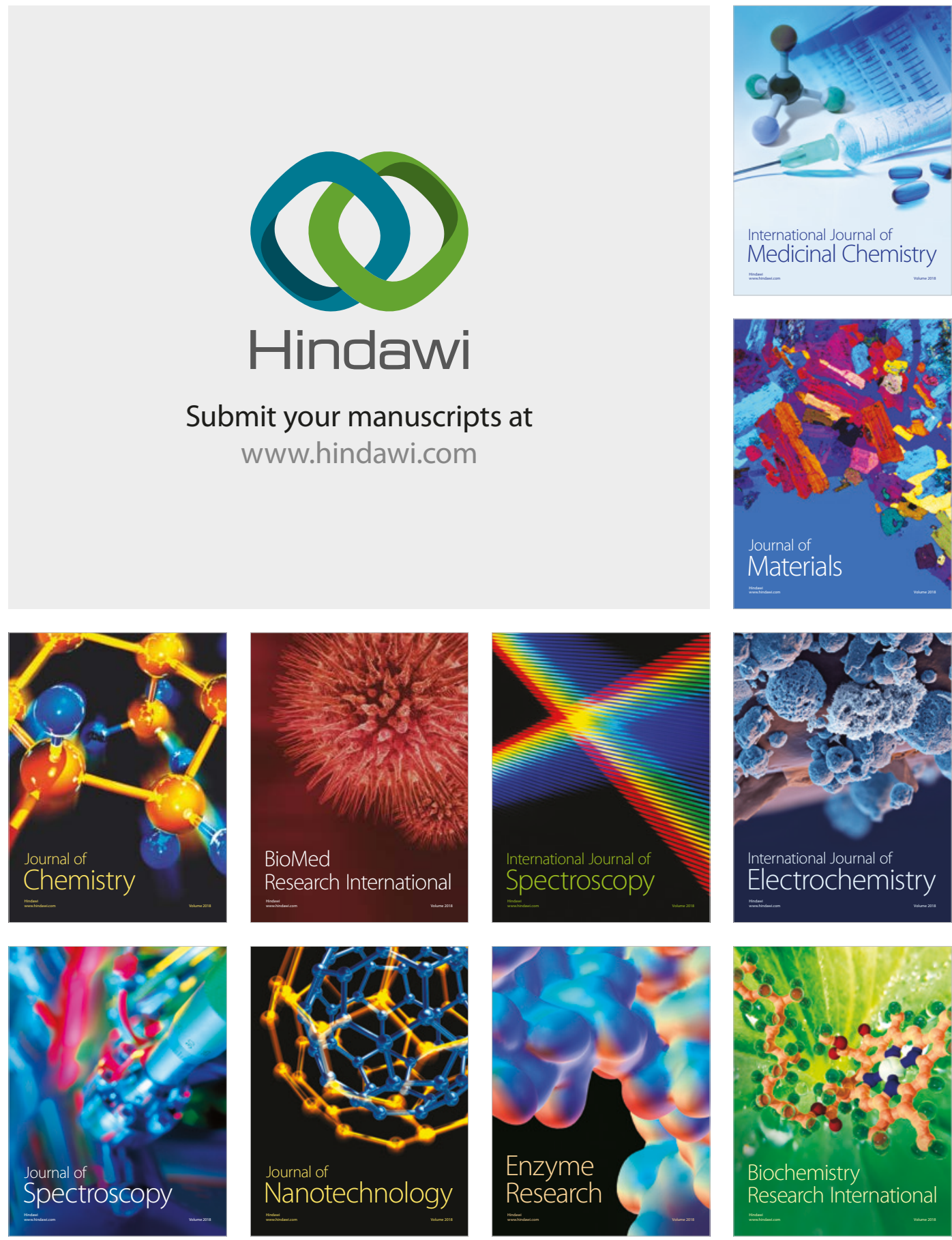
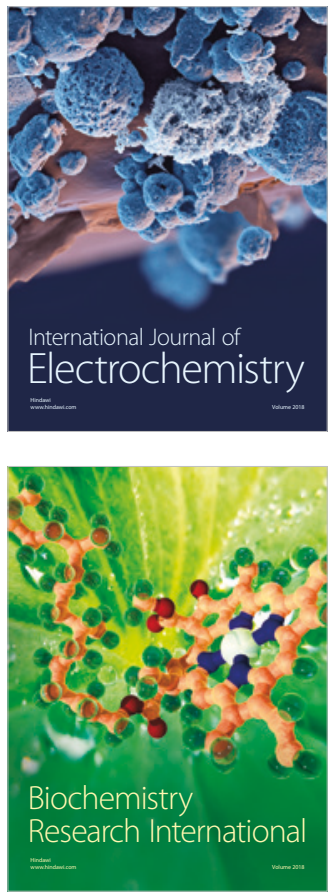